\title{
Aporte del análisis de colecciones a las modalidades de aprovisionamiento: El caso de los sitios cantera-taller del Valle de Yocavil (Noroeste Argentino)
}

\author{
Juan Pablo Carbonelli \\ CONICET. Museo Etnográfico, Universidad de Buenos Aires (UBA), Estados Unidos $33873^{\circ}$ A Lanús Oeste, \\ Argentina. Email: juanp.carbonelli@gmail.com
}

\begin{abstract}
Resumen:
En este trabajo nos proponemos analizar y comparar entre sí cuatro colecciones de artefactos, las cuales provienen de sitios de superficie situados al sur de los valles Calchaquíes. Los sitios canterataller de Ampajango, El Bordo y Los Zazos han sido estudiados con anterioridad y parte de sus piezas se encuentran en el Museo de Ciencias Naturales de la Universidad Nacional de La Plata y el Museo de Antropología de la Universidad Nacional de Rosario, ambos en la Argentina. En este trabajo ofrecemos una revisión y una nueva interpretación de dichos conjuntos artefactuales. Desde el aspecto metodológico, los análisis estadísticos nos permitieron reconocer los sesgos que construyeron dichas muestras. Los test nos indican que hay una menor proporción de artefactos con barniz del desierto en las colecciones de lo que se ajustaría a la realidad. Esto incide básicamente en la ausencia de casos de reclamación, evidentes en las diferencias de barniz entre lascados. Por otra parte los análisis de diversidad nos advierten que en las colecciones hay una sobre-representación de los artefactos formatizados, lo que dificulta la descripción de la secuencia de producción. Partiendo de dichos sesgos, mediante el análisis tecno-morfológico caracterizamos la producción lítica de los sitios cantera-taller. En términos generales, los mismos presentan una tecnología expeditiva compuesta por núcleos amorfos y bifaces parciales, que luego fueron utilizados como núcleos.
\end{abstract}

Palabras clave: Valle de Yocavil; cantera-taller; colecciones; cazadores-recolectores; bifaces

\section{Introducción}

El conocimiento sobre sociedades cazadoras-recolectoras en las zonas de los valles mesotermales del noroeste argentino, tuvo que superar desde el comienzo de las investigaciones el obstáculo de la carencia de una cronología que pudiera contextualizar los hallazgos. Las primeras intervenciones fueron efectuadas por el doctor Cigliano y equipo, quienes partieron desde la ciudad de Rosario con la premisa explícita de ubicar yacimientos áceramicos (Cigliano et al. 1962).

En la localidad arqueológica de Ampajango, Cigliano et al. (1962) definieron una industria lítica, conformada principalmente por bifaces, unifaces, raspadores y raederas. Todos estos materiales tenían un gran tamaño y morfológicamente fueron descriptos como toscos o primitivos (Cigliano 1961). Detectada en el sitio Ampajango, esta "industria” se

Published by the School of History, Classics and Archaeology, University of Edinburgh ISSN: 2055-0472. URL: http://journals.ed.ac.uk/lithicstudies/

This work is licensed under a Creative Commons Attribution 2.5 UK: Scotland License. 
habría extendido por el resto de los Valle Calchaquíes, llegando hasta Río Grande de Jujuy (Jujuy) (Cigliano 1964; 1968).

En el caso específico de la cantera-taller Ampajango, al no poder ubicar material en estratigrafía, Cigliano et al. (1962) utilizaron dos criterios para establecer un marco cronológico. En primer término, los investigadores consideraron que la erosión que formó la terraza y dejó al descubierto la depresión sobre la cual se encontraba la cantera-taller no podría haberse producido en el período holocénico (10000 AP). Por lo tanto, la antigüedad de la cultura “Ampajangense” sería superior a los diez mil años. En segundo lugar, los investigadores le atribuyeron a la técnica de manufactura de los artefactos una temporalidad.

Existía otra cualidad de los artefactos, la cual no fue considerada por las primeras exploraciones de estos sitios de superficie. Gran parte del material se halla cubierto por un tipo de corrosión denominada "barniz del desierto". Un grupo de geólogos del Centro de Estudio de Regiones Secas retomó este vértice de la investigación. Analizaron tanto los materiales que provenían de sus trabajos de campo como de las colecciones de la Universidad Nacional de La Plata. Particularmente, en el caso del barniz del desierto propusieron que su formación se debía a un proceso especialmente químico en el cual, el principal agente meteorizante es el agua (García Salemi \& Durando 1985).

En los últimos años, los trabajos en la Quebrada de Amaicha han posibilitado un nuevo giro en la interpretación sobre estos sitios de superficie. Somonte (2009: 4-7) tomó como eje estructural de sus investigaciones las diversas estrategias de aprovisionamiento de las sociedades que ocuparon los paisajes de la Quebrada de Amaicha a partir del Holoceno Medio. Los estudios en sitios a cielo abierto, Planchada La Puntilla y Río Las Salinas 1 y 2 dan cuenta de una multiplicidad de eventos: abastecimiento de materia prima, manufactura de artefactos líticos y realización de actividades específicas (Baied \& Somonte 2012). Baied y Somonte (2012) sugieren que diversos grupos humanos a lo largo de milenios han asistido a estas canteras para proveerse de materia prima y confeccionar instrumentos de corte expeditivo. Una característica de estos conjuntos artefactuales es la evidencia de reclamación, constatada a partir de la diferencia de barniz en los negativos de lascados de los instrumentos, lo cual permitió considerar a parte de estos sitios como fuentes terciarias (sensu Church 1995).

La información generada en espacios vecinos constituyó una iniciativa para retomar los trabajos en el sitio cantera-taller localizado en Ampajango (Carbonelli 2013a), sobre el que se basó Cigliano (Cigliano et al. 1962), para definir la Industria Ampajanguense. Dada la ausencia de material disponible para fechar por métodos convencionales y los buenos resultados de las dataciones por Varnish Microlamination (VML) en sitios "ampajanguenses" de la Quebrada de Amaicha (Somonte \& Baied 2013), decidimos datar desechos de talla con presencia de barniz en la cantera-taller de Ampajango. En este caso, las dataciones por VML señalan que la edad mínima de la superficie donde se encontraron dichos artefactos es de 9400 años AP, identificándose hasta diez eventos húmedos en la secuencia total de microlaminaciones (Carbonelli 2013b: 216-222).

Por otra parte, la información recabada desde la tecnología nos permitió sostener que desde dicha edad mínima, el sitio Ampajango funcionó como una cantera-taller. Esta fue utilizada por distintas comunidades humanas, las cuales aprovecharon los bloques con andesitas, los artefactos formatizados y los desechos generados en el tiempo. Las evidencias de procesos de reciclaje y solapamiento de filos, principalmente sobre bifaces y otros artefactos formatizados con arista sinuosa (Carbonelli 2013b: 212-214) indican que existió una diacronía en las actividades y una intención en concebir a estos espacios como un punto de retorno.

Sin embargo, para poder caracterizar de manera adecuada las estrategias de aprovisionamiento y el uso que distintas sociedades han hecho de los espacios cantera-taller 
denominados ampajanguenses, necesitamos tener una mirada regional. Una solución factible sería revisitar los sitios descritos por Cigliano (1968). No obstante, como marcan sus publicaciones, parte de las piezas recolectadas se encuentran en museos de las diferentes universidades donde dicho investigador guardaba su pertenencia institucional. Dado que el registro arqueológico ha sido alterado y no podemos volver a observar su composición original, nos preguntamos: ¿Qué información nos puede aportar el estudio de las colecciones ampajanguenses, en referencia a las estrategias de aprovisionamiento? ¿Nos permitirán observar procesos complejos como la reclamación? ¿Qué rol ocuparon estos espacios a escala regional en las decisiones de aprovisionamiento y explotación de la materia prima?

\section{Materiales y metodología}

$\mathrm{T}$ Los materiales a analizar provienen de colecciones de museo, integradas en su totalidad por artefactos líticos cuya procedencia son sitios cantera-taller situados al sur de los valles Calchaquíes. Precisamente, las muestras corresponden al Museo de Ciencias Naturales de la Universidad Nacional de La Plata (Mus.C.N UNLP) y al Museo de Antropología de la Universidad Nacional de Rosario (Mus. Ant. UNR) (Carbonelli 2013b: 233-216). En el primer caso, la colección se halla subdividida: a) sitio cantera-taller Ampajango, localizado en el valle de Santa María, b) sitio Los Zazos, en Amaicha del Valle (ambos analizados por Durando et al. 1986) y c) sitio El Bordo, en Entre Ríos (Figura 1). En el segundo caso, la colección se encuentra conformada únicamente por los artefactos recolectados del sitio cantera-taller Ampajango.

\subsection{Base regional de recursos líticos}

Antes de comenzar a discutir los procesos de producción lítica observados en los sitios cantera-taller, es imprescindible dar a conocer la estructura regional de recursos líticos de este sector de los valles Calchaquíes. Para ello nos valemos de nuestras propias prospecciones en distintos sectores del valle, las cuales nos permitieron detectar fuentes potenciales y efectivas de materias primas (Carbonelli 2012). A esto debemos agregar la información aportada por los cortes petrográficos, a través de los cuales pudimos caracterizar mineralógicamente las materias primas e identificarlas.

La unidad de análisis espacial es el Valle de Yocavil, el cual se encuentra localizado en el sector norte de las Sierras Pampeanas, ocupando el extremo nor-oriental de la provincia de Catamarca (Departamento de Santa María) y el ángulo noroeste de la provincia de Tucumán (Departamento de Tafí). Es atravesado por el meridiano $66^{\circ}$ al norte y al sur limita con los paralelos $26^{\circ}$ y $27^{\circ}$. Limita hacia el este con las Sierras del Aconquija y hacia el oeste con las Sierras del Cajón.

En cuanto a las rocas volcánicas, existen dos variedades de rocas andesitas detectadas en el Valle de Yocavil: una gris de textura afanítica/porfídica (andesita g) y una oscura (andesita b) de textura netamente afanítica. La escasa cantidad de fenocristales bajo observación macroscópica de la andesita b, le otorga una calidad superior con respecto a la variedad gris. Ésta frecuentemente presenta inclusiones de cuarzo y pequeñas rajaduras.

En el caso de la andesita g abunda en forma de rodados en los ríos que nacen en el Aconquija y desaguan en el Río Santa María. También se constata la presencia de este recurso lítico en los glacis de piedemonte, donde se distribuye en forma de grandes conglomerados conformado por bloques y rodados. Finalmente, debemos mencionar como fuente la depresión donde se ubica la cantera-taller de Ampajango. Allí encontramos una distribución concentrada de bloques de andesita, cubierto por barniz de rocas. En el caso de la andesita b, si bien se constató la presencia de una fuente efectiva de dicha variedad en el sector 
pedemontano del valle de Yocavil, el espacio donde se encontró la mayor frecuencia de esta variedad fue en la localidad de Ampajango, sobre el depósito fluvial del río homónimo.

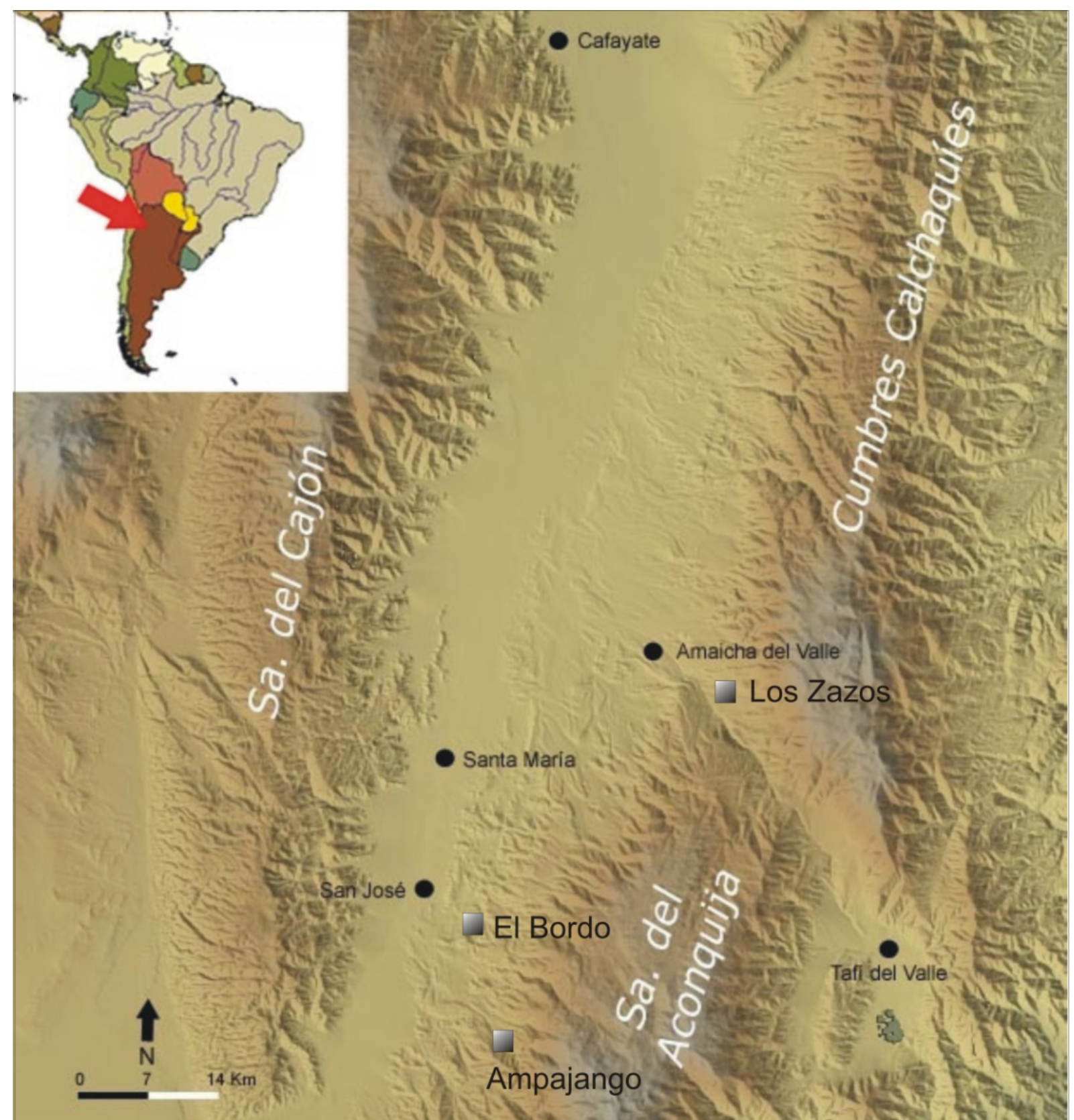

Figura 1. Localización del sitio Ampajango y el lugar de procedencia de la muestras (modificada de Spano 2011).

Figure 1. Geographical location of Ampajango site and place of origin of the samples (modifidied of Spano 2011).

En lo que respecta al cuarzo, este es uno de los recursos con mayor disponibilidad de los que se registra su uso en sitios arqueológicos (Carbonelli 2012). Su calidad para la talla es de regular a mala, dada su excesiva dureza. Existen fuentes primarias (sensu Nami 1992) constituídas por afloramientos, diques y filones presentes en la Formación Andalhuala, en la Formación Yayasmayo y en el Basamento Cristalino (Ruiz Huidobro 1972: 10). No obstante, consideramos que las canteras principales de estos recursos son los rodados presentes en los lechos de los ríos que atraviesan el valle. La fuente de cuarcita, de buena calidad para la talla, no ha sido localizada en nuestras prospecciones. Sin embargo, se han detectado bloques de 
cuarcita en la zona del Cerro Mendoncino, dentro del sector montañoso de las Sierras del Cajón.

Por último, debemos mencionar las rocas metamórficas. Hemos englobado en este conjunto de rocas a: los esquistos, gneises, filitas y pizarras. En los glacis de Ampajango, Andalhuala y en la zona de piedemonte de Caspinchango se distinguen fácilmente en el paisaje grandes bloques de rocas metamórficas, los cuales alcanzan los tres metros de largo. Consideramos que por su tamaño, se trata de fuentes primarias, es decir afloraciones. En cuanto a las fuentes secundarias, observamos que sobre el lecho del río Entre Ríos, cercano al sitio El Bordo, predominan estos recursos líticos. Los mismos se encuentran disponibles en forma de grandes bloques o rodados de hasta $40 \mathrm{~cm}$ de diámetro, tanto en las cárcavas de los cauces secos, como en los lechos de los ríos. Su calidad para la talla, por las inclusiones de cuarzo y granates sumada a la ausencia de fractura concoidal, es muy mala. No obstante, su textura abrasiva la torna útil para las actividades de molienda.

En referencia a la Quebrada de Amaicha, donde se encuentra el sitio Los Zazos, los trabajos de Somonte y Baied (2011) señalan que son las distintas variedades de andesitas las que se encuentran mayormente disponibles en el paisaje, mientras que otros recursos como cuarcitas y sílices, exhiben una distribución más acotada. Los autores marcan una coincidencia con nuestro trabajo al indicar que las rocas metamórficas son abundantes.

El único recurso no inmediatamente local o local lejano (sensu Civalero \& Franco 2003), ubicado entre los 20 y $40 \mathrm{~km}$ de las canteras-taller es la madera silicificada encontrada en la cantera-taller de Los Zazos, la cual podría provenir de la localidad de Tiu Punco (Somonte 2009: 123).

\subsection{Análisis tecno-morfológico y estadístico}

Si queremos conocer de qué forma las canteras-taller participaron de la organización de la tecnología de los cazadores-recolectores de las región, debemos determinar las etapas de la secuencia de producción que se encuentran presentes (Bellelli 2005). Las piezas que conforman la muestra fueron analizadas a partir del enfoque tecno-morfológico y morfológico funcional de Aschero (1975; 1983). En primer término, distinguiremos dentro del conjunto lítico las clases tipológicas, las cuales permiten diferenciar a los artefactos en núcleos, desechos de talla, artefactos formatizados y artefactos con rastros complementarios (Aschero \& Hocsman 2004). En el caso de los desechos se evaluaron las siguientes características: materias primas, tamaño, presencia de corteza y tipo de lasca. En el caso de los núcleos se evaluó la designación morfológica de los ejemplares, su materia prima e intensidad en la reducción de los núcleos.

Existieron dos aspectos llamativos que se desprenden de nuestras primeras observaciones, al comparar los conjuntos instrumentales del sitio Ampajango con las colecciones de los museos. Uno de ellos es el mayor porcentaje de artefactos cubiertos por barniz en las piezas recolectadas por nosotros en nuestros trabajos de campo (Carbonelli 2013b: 186). Esta variable adquiere particular relevancia por cuanto a mayor cantidad de artefactos con presencia de barniz existe una mayor probabilidad de detectar casos de reclamación; es decir de diferencia temporal entre series distintas de lascados. La presencia de artefactos reclamados, permitiría considerar los espacios de donde provienen las muestras, como fuentes terciarias.

Con la intención de detectar si existieron, o no, sesgos en la recolección de las piezas que conformaron las colecciones efectuaremos una comparación entre éstas y los artefactos recolectados en nuestros trabajos de campo (Carbonelli 2013a). Para ello efectuaremos análisis estadísticos, trabajando con variables nominales (como la presencia o ausencia de barniz). En este caso, compararemos cinco conjuntos (el sitio Ampajango, la colección 
Ampajango del Mus. Ant. UNR, y las colecciones de los sitios Ampajango, Los Zazos y El Bordo del Mus. C.N. UNLP), a partir de la frecuencia observada de la variable de interés (presencia o ausencia de barniz).

Efectuaremos la comparación a través del análisis de Chi $^{2}$. Es de nuestro interés contrastar la hipótesis nula de que no hay diferencias entre las proporciones de artefactos con barniz en los cinco conjuntos descritos. Por consiguiente la hipótesis alternativa es que existe alguna diferencia, es decir que las proporciones no son iguales y responden a una explicación del tipo conductual en la conformación del registro arqueológico o a un sesgo en la recolección.

El segundo problema que detectamos es el desbalance existente entre la elevada frecuencia de artefactos formatizados en detrimento de los desechos de talla y núcleos (Tabla 1). Con el objetivo de abordar de manera cuantitativa la diversidad de cada conjunto, compararemos las clases tipológicas de cada uno de los conjuntos entre sí. La diversidad es una forma de medir la variación ya que describe atributos de una población, compuesta por clases (atributos) que varían en su frecuencia (riqueza), lo que nos permite discutir cuestiones estructurales de la muestra. En definitiva buscamos hallar un patrón en nuestras observaciones, explicando las diferencias entre los conjuntos bajo la forma de un proceso. Para explorar la riqueza utilizaremos indicadores que no son demasiado sensibles al tamaño de las muestras, como puede ser el Índice de Dominancia de Simpson y el Eveness. El Índice de Dominancia de Simpson muestra la medida en la que una clase particular, predomina en la muestra, por lo cual puede ser utilizado para diferenciar entre conjuntos. Por otro lado, el índice de homogeneidad (Eveness) indica el grado en que cada una de las clases tiene una representatividad similar.

Tabla 1. Materias primas por clases tipológicas en las colecciones de museos. Referencias: Af: artefactos formatizados; Nc: núcleos; De: desechos; Anf: artefactos no formatizados; FNRC: filo natural con rastros complementarios; UNR: Universidad Nacional de Rosario; UNLP: Universidad Nacional de La Plata.

Table 1. Raw material by typological classes in museum collections. References: Af: knapped tool; Nc: cores; De: debitage; Anf: non-knapped tool; FNRC: Natural working edges; UNR: National University of Rosario; UNLP: National University of La Plata.

\begin{tabular}{|c|c|c|c|c|c|c|c|c|c|c|c|c|c|c|c|}
\hline \multirow[t]{2}{*}{ Materias Primas } & \multicolumn{4}{|c|}{$\begin{array}{c}\text { Sitio Ampajango } \\
\text { UNR }\end{array}$} & \multicolumn{5}{|c|}{$\begin{array}{c}\text { Sitio Ampajango } \\
\text { UNLP }\end{array}$} & \multicolumn{3}{|c|}{$\begin{array}{c}\text { Sitio Los Zazos } \\
\text { UNLP }\end{array}$} & \multicolumn{3}{|c|}{$\begin{array}{c}\text { Sitio El Bordo } \\
\text { UNLP }\end{array}$} \\
\hline & Af & & & Anf & Af & Nc & $\mathrm{De}$ & Anf & Fnrc & Af & $\mathrm{Nc}$ & & Af & $\mathrm{Nc}$ & $\mathrm{De}$ \\
\hline Andesita g & 42 & 15 & 69 & 1 & 4 & 1 & 26 & - & - & 258 & 34 & 73 & 12 & 1 & 20 \\
\hline Cuarcita & - & 1 & - & 1 & - & - & - & - & - & - & - & - & 1 & 1 & 1 \\
\hline Anfibolita & - & - & - & - & - & - & 1 & - & - & - & - & - & - & - & - \\
\hline Andesita $b$ & - & - & - & - & 3 & 1 & 1 & 1 & - & - & - & - & - & - & 1 \\
\hline Metamórfica & - & - & - & - & 8 & - & 57 & - & 1 & - & - & - & - & - & - \\
\hline Madera silificada & - & - & - & - & 2 & - & - & - & - & - & - & - & - & - & - \\
\hline Indeterminada & - & - & - & - & - & - & - & - & - & 3 & - & - & - & - & - \\
\hline Subtotal & 42 & 16 & 69 & 1 & 17 & 2 & 85 & 1 & 1 & 261 & 34 & 73 & 13 & 2 & 22 \\
\hline Totales & & 128 & & & & & 106 & & & & 368 & & & 37 & \\
\hline
\end{tabular}

\section{Resultados}

\subsection{Análisis estadístico}

En la Tabla 2 exhibimos la tabla de contingencia, con la frecuencia de materiales con barniz de las muestras (del sitio y de las colecciones aquí analizadas). Las diferencias se vuelven más notorias al estandarizar dichos valores en porcentajes: mientras que en el sitio 
Ampajango, el porcentaje de piezas con barniz alcanza el 40\%, en las colecciones del Mus. Ant. UNR y el Mus. C.N. UNLP se encuentran entre el 5 y el $20 \%$ respectivamente. A partir de estos datos efectuamos el análisis del $\mathrm{Chi}^{2}$ (utilizando el programa PAST), y comparamos la cantidad de pátina/ausencia entre las muestras (Tabla 3).

Tabla 2. Presencia y ausencia de barniz. Artefactos del sitio Ampajango y colecciones. Referencias: Colec: colecciones; UNR: Universidad Nacional de Rosario; UNLP: Universidad Nacional de La Plata.

Table 2. Presence and absence of rock varnish. Ampajango site artifacts and collections. References: Colec: Collections; UNR: National University of Rosario; UNLP: National University of La Plata.

\begin{tabular}{lccc}
\hline Colecciones & Presencia & Ausencia & $\mathbf{n}$ \\
\hline Ampajango & 77 & 100 & 177 \\
Colec. Ampajango UNR & 27 & 101 & 128 \\
Colec. UNLP Los Zazos & 114 & 254 & 368 \\
Colec. UNLP Ampajango & 3 & 103 & 106 \\
El Bordo & 1 & 36 & 37 \\
Total & 222 & 594 & 816 \\
\hline
\end{tabular}

Tabla 3. Resultados de $\mathrm{Chi}^{2}$.

Table 3. Chi $^{2}$ results.

Presencia vs. Ausencia

One constraint

\begin{tabular}{lrr}
\hline N1: & 222 \\
N2: & 594 \\
Deg. freedom: & 4 & \\
Chi $^{2}:$ & & 71,816 \\
Monte Carlo p(same): & 0,0001 & \\
\hline
\end{tabular}

A través de los resultados observamos que se contrastó la hipótesis nula. Los valores altos de $\mathrm{Chi}^{2}$ indican que hay diferencias significativas entre las distintas colecciones, particularmente porque el conjunto artefactual que recolectamos de Ampajango presenta una mayor proporción de pátina (estadísticamente significativa) que el resto de las colecciones.

Trataremos de clarificar dicha situación, a través de la Figura 2 que da a conocer las distribuciones esperadas para todos los conjuntos, de acuerdo a los valores residuales. Los rectángulos en rojo indican que la distribución es mayor a la esperada por azar, mientras que los tonos grises indican que se encontraron menos especímenes de los esperados. Por ejemplo, en el caso del sitio Ampajango, la presencia de artefactos con pátina es mayor a lo esperado, mientras que hay mucho menos artefactos sin pátina que si las frecuencias se distribuyeran uniformemente entre los casos.

Dos casos sobresalen del resto: tanto en la colección El Bordo, como en la Colección Ampajango de la UNLP, existen muchos menos artefactos con pátina de lo que determinaría el azar. Esto resalta el sesgo en la recolección de las muestras que conformaron la colección de los museos. Es factible pensar a través de estos resultados, que muchas piezas con pátinas fueron descartadas o ni siquiera contempladas en el momento de la recolección.

Profundizando en esta problemática, nos interesó registrar la diversidad de los conjuntos instrumentales analizados, en referencia a su composición. En otras palabras, sobre cómo se distribuían las clases tipológicas en cada muestra (Tabla 1). 

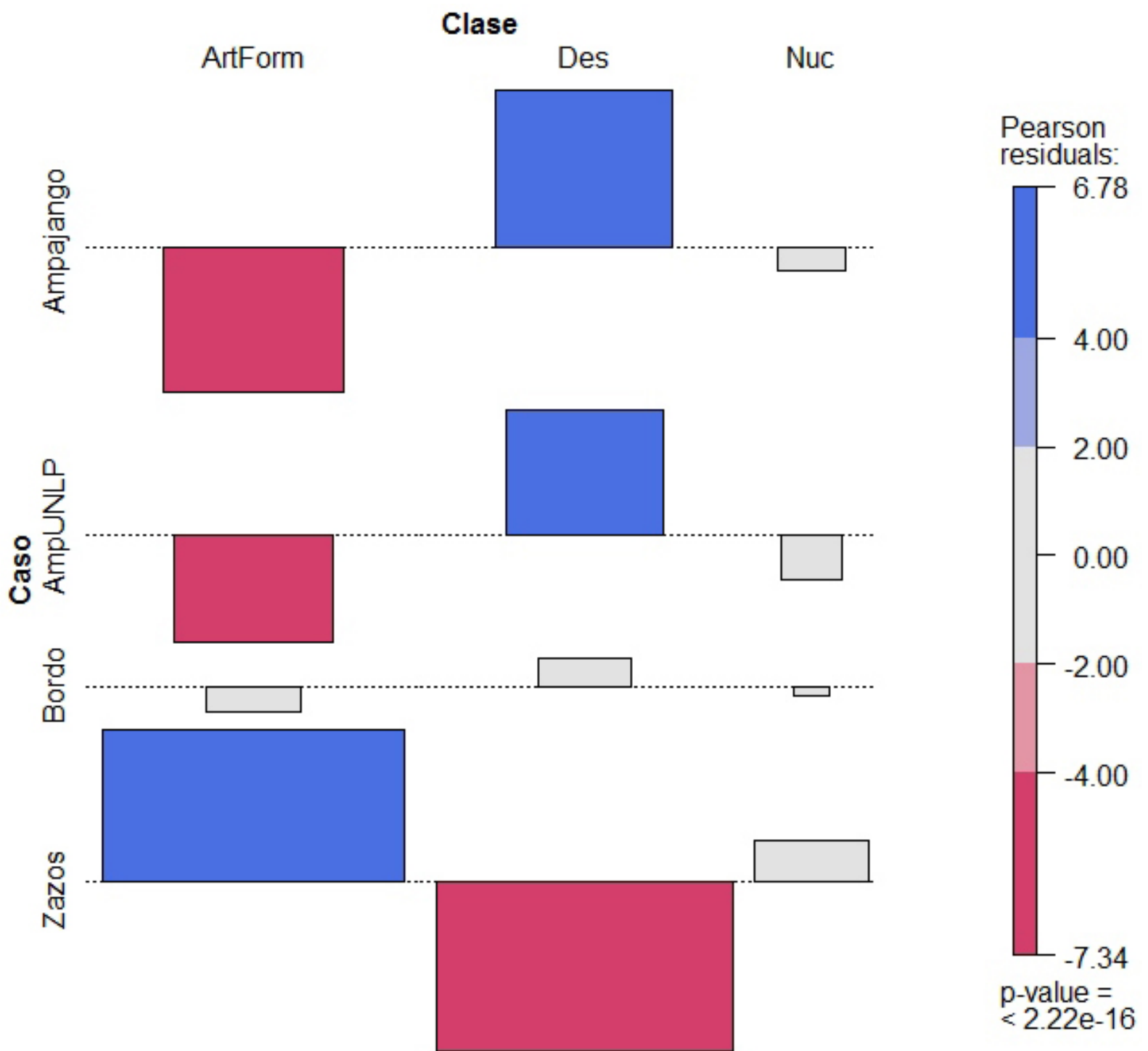

Figura 2. Distribuciones esperadas de barniz en los conjuntos artefactuales. Referencias: Zazos: Colección Los Zazos del Mus. C.N.UNLP; Rosario: Colección de Ampajango del Mus. Ant. UNR; ColAmp: Colección de Ampajango del Mus. C.N. UNLP; Bordo: colección El Bordo del Mus. C. N. UNLP; Amp: Sitio Ampajango.

Figure 2. Expected distributions of rock varnish in artifactual sets. References: Zazos: Collection of Los Zazos from Natural Science Museums UNLP; Rosario: Collection of Ampajango Anthropology Museum UNR; ColAmp: Collection of Ampajango Natural Science Museums UNLP; Bordo: Collection of El Bordo Collection of Ampajango Anthropology Museum UNR; Amp: Site Ampajango.

Al observar la Tabla 4, detectamos que los mayores índices de dominancia (Dominance_D) se encuentran en el sitio Ampajango y en la colección Ampajango del Museo de Ciencias Naturales de la UNLP. La clase tipológica que domina la muestra son los desechos de talla, dato esperable para sitios canteras-taller. En contraposición, el resto de las muestras exhiben índices de homogeneidad (Evenness_e ${ }^{\wedge} \mathrm{H} / \mathrm{S}$ ) altos, donde las tres clases tipológicas se distribuyen de forma más equitativa. Un caso paradigmático es la colección Los Zazos del Museo de Ciencias Naturales de la UNLP. En este sentido, la frecuencia de artefactos formatizados $(n=261)$ es alta en comparación al total de la muestra: consideramos que aquí no se efectuó una recolección sistemática, pues hay una mayor proporción de instrumentos de lo esperable para sitios cantera-taller. 
Tabla 4. Índices de diversidad del sitio Ampajango y coleccciones. Referencias: UNR: Universidad Nacional de Rosario; UNLP: Universidad Nacional de La Plata.

Table 4. Indices of diversity from Ampajango site and collections. References: UNR: National University of Rosario; UNLP: National University of La Plata.

\begin{tabular}{lccccc}
\hline & Ampajango & $\begin{array}{c}\text { C. Ampajango } \\
\text { UNR }\end{array}$ & $\begin{array}{c}\text { C. Los Zazos } \\
\text { UNLP }\end{array}$ & $\begin{array}{c}\text { C. Ampajango } \\
\text { UNLP }\end{array}$ & $\begin{array}{c}\text { C. El Bordo } \\
\text { UNLP }\end{array}$ \\
\hline Taxa_S & 3 & 3 & 3 & 3 & 3 \\
Individuals & 132 & 127 & 368 & 104 & 37 \\
Dominance_D & 0,7446 & 0,4204 & 0,5509 & 0,6951 & 0,4799 \\
Simpson_1-D & 0,2554 & 0,5796 & 0,4491 & 0,3049 & 0,5201 \\
Shannon_H & 0,5018 & 0,9584 & 0,7846 & 0,5369 & 0,8343 \\
Evenness_e^H/S & 0,5506 & 0,8692 & 0,7305 & 0,5702 & 0,7678 \\
\hline
\end{tabular}

En síntesis, estos test nos señalan en primer lugar que hay una menor proporción de artefactos con barniz del desierto en las colecciones de lo que se ajustaría a la realidad. Si contáramos con mayores porcentajes de piezas con barniz, hubieran aumentado las probabilidades de detectar casos de reclamación, evidentes en las diferencias de barniz entre lascados (Somonte 2009: 11). En segundo lugar, los análisis de diversidad nos advierten que en las colecciones hay una sobre-representación de los artefactos formatizados. Esto nos indica que las clases menos representadas son los desechos de talla y núcleos, razón por la cual, faltan elementos para describir adecuadamente las secuencias de producción (Carbonelli 2013b: 302-307).

\subsection{Análisis tecno-morfológico de las colecciones}

Los análisis estadísticos nos obligan a ser cautos a la hora de analizar las muestras de los sitios de superficie, que provienen de las colecciones de los museos. No obstante, consideramos necesario profundizar nuestra mirada sobre la composición artefactual debido a que, aún con los sesgos detectados, son el único testimonio existente de estos sitios.

En la Tabla 5 exhibimos las características de los núcleos y desechos de talla. En este caso nos abocaremos especialmente a las muestras provenientes de las colecciones, ya que la composición artefactual del sitio Ampajango ya fue comentado en otro trabajo (Carbonelli 2013a).

La mayoría de los núcleos presentes en las colecciones, con excepción de los bifaciales y bipiramidales, corresponden a la clasificación de núcleos amorfos. Dicha clasificación obedece a la ausencia de plataformas auxiliares o de mantenimiento, que permitan reactivar la plataforma principal ante problemas de manufactura. No encontramos tampoco frentes auxiliares y lascados de mantenimiento que posibilitan readecuar el diseño y mantener la continuidad en el aprovechamiento (Paulides 2006).

Precisamente es en los núcleos bifaciales donde encontramos, en promedio, el mayor número de extracciones, seguidos por los núcleos bipiramidales. Consideramos que existe un mayor aprovechamiento de los núcleos bifaciales y bipiramidales sobre el resto, dado que los primeros exhiben extracciones más largas y anchas. Finalmente, al observar la frecuencia de núcleos agotados (Carbonelli 2013b: 235), obtenemos que los núcleos bifaciales sobresalen del resto, como una consecuencia de una búsqueda por extraer la mayor cantidad de lascas de los mismos. 
Tabla 5. Atributos de los desechos de talla y núcleos de las colecciones. Referencias: Amp.UNR: Ampajango Universidad Nacional de Rosario; Amp.UNLP: Ampajango Universidad Nacional de La Plata; Pir. Irreg: Piramidales irregulares; Disc. Irreg: Discoidales irregulares; Ls: Lascados; Pir. Reg: Piramidales regulares; Ls. ang: Lascas angulares; Ls. reduc. bif: lascas de reducción bifacial; Ls. no dif: lascas no diferenciadas; Ls. Reac. Nuc: Lascas de reactivación de núcleo; Ls. de Reac: Lascas de reactivación.

Table 5. Debitage and collections core attributes. References: Amp.UNR: Ampajango National University of Rosario; Amp. UNLP: Ampajango National University of La Plata; Pir. Irreg: irregular pyramidals; Disc.Irreg: irregular discoidals; Ls. Aislados: isolated flakes; Pir.Reg: regular pyramidals; Ls. Ang: angular flakes; Ls. Reduc. Bif: bifacial reduction flakes; Ls no dif: Undifferentiated flakes; Ls. Reac.Nuc: Rejuvenation core flakes; Ls. de Reac: rejuvenation flakes.

\begin{tabular}{lccccc}
\hline $\begin{array}{l}\text { Tipos de } \\
\text { Núcleos }\end{array}$ & $\begin{array}{c}\text { Sitio } \\
\text { Ampajango } \\
\text { UNR }\end{array}$ & $\begin{array}{c}\text { Sitio } \\
\text { Ampajango } \\
\text { UNLP }\end{array}$ & $\begin{array}{c}\text { Sitio Los } \\
\text { Zazos } \\
\text { UNLP }\end{array}$ & $\begin{array}{c}\text { Sitio El Bordo } \\
\text { UNLP }\end{array}$ & Total \\
\hline Bifaciales & 3 & 1 & 18 & - & 22 \\
Pir. Irreg & 7 & 1 & 6 & 1 & 15 \\
Poliédricos & 1 & - & 1 & 1 & 3 \\
Disc. Irreg & 2 & - & 1 & - & 3 \\
Ls. Aislados & 3 & - & - & - & 3 \\
Pir. Reg & - & - & 5 & & 5 \\
Bipiramidales & - & - & 3 & & 3 \\
& & & & & \\
Tipos de Lascas & & 4 & 20 & - & 5 \\
Ls ang & 25 & 14 & 3 & - & 8 \\
Ls. reduc. Bif & 3 & 7 & - & - & 5 \\
Ls no dif & 1 & 1 & 7 & - & 5 \\
Ls. Nodular & 1 & - & 3 & & \\
Ls primaria & 2 & - & 5 & & \\
Ls. Reac. Nuc. & - & - & - & & \\
Ls. de Reac & - & & & & \\
\hline
\end{tabular}

Como se desprende de la tabla 5, existe un predominio de las lascas internas sobre las externas. La sub-representación de las primeras etapas de la secuencia de producción puede ser explicada por lo sesgos mencionados en los análisis estadísticos. En contraposición, la elevada frecuencia de lascas internas, como las angulares y de reducción bifacial, señalan que estarían sobre-representadas las tareas de formatización y regularización de filos en estos espacios de producción lítica. No obstante, no debemos pasar por alto que los desechos indiferenciados (entre los que no se puede diferenciar la cara dorsal y ventral, con bordes irregulares) frecuentemente se desprenden de la talla por percusión, como resultado de la reducción de nódulos o extracción de formas base (Bellelli 2005).

En referencia a los tipos de talones registrados en las lascas enteras y fracturadas con talón, si bien la muestra es muy pequeña, refleja la misma tendencia en la producción. Los talones lisos (78,96\%) dan cuenta de las actividades de percusión directa en el sitio y se encuentran mayormente relacionados con la manufactura de instrumentos (Valverde 2003) mientras que los porcentajes de talones diedros $(7,89 \%)$ y facetados $(2,63 \%)$ aunque bajos, dan cuenta de actividades asociadas a la reducción bifacial. Finalmente, los valores de talones corticales (10,52\%), consideramos que se encuentran unidos a las actividades de reducción primaria.

Todas las materias primas representadas en las categorías núcleos y desechos son de origen local. Nos referimos concretamente a las dos variedades de andesitas, la cuarcita y las rocas metamórficas. Es en estos casos donde podemos inferir tareas de extracción de formas bases y formatización de instrumentos en el sitio. En cambio, en el caso de la madera 
silicificada, que sólo se encuentra presente entre los artefactos formatizados, sostenemos el ingreso de los mismos a las canteras-taller una vez manufacturados.

$\mathrm{Al}$ observar la frecuencia de rocas metamórficas en las distintas clases tipológicas llama la atención su ausencia completa entre los núcleos, lo cual contrasta con sus frecuencias entre los desechos de talla y artefactos formatizados. Nuestro conocimiento en otros sitios del valle, como Soria 2, donde también se encontraron artefactos formatizados y desechos de esta materia prima (Carbonelli 2011) permite sostener que en estas rocas la reducción primaria se efectuó directamente sobre los nódulos/rodados/lajas.

En el análisis de los artefactos formatizados, hemos distinguido los grupos tipológicos que agrupan a los artefactos bifaciales (Aschero \& Hocsman 2004; Hocsman 2006: 93-101) de otros grupos tipológicos (Tabla 6). Por otra parte, hemos discriminado la presencia de artefactos formatizados con filos complementarios, ya que ésto puede ser considerado evidencia de diseños multifuncionales (Elías 2010: 154), o a estrategias tecnológicas tendentes a maximizar las materias primas (Escola 2000: 180)

Tabla 6. Grupos tipológicos en las colecciones de museos. Referencias: Esb. Piez. Bif: esbozos de piezas bifaciales; Fbas: filo bifacial de arista sinuosa; Artefactos com. Artefactos compuestos; Amp.UNR: Ampajango Universidad Nacional de Rosario; Amp.UNLP: Ampajango Universidad Nacional de La Plata.

Table 6. Typological groups in museum collections. References: Esb. Piez.Bif: outlines of bifacial pieces; Fbas: bifacial edge with sinuous ridges; Amp.UNR: Ampajango National University of Rosario; Amp. UNLP: Ampajango National University of La Plata.

\begin{tabular}{lcccccccc}
$\begin{array}{c}\text { Grupos } \\
\text { tipológicos }\end{array}$ & $\begin{array}{c}\text { Colección } \\
\text { Amp. UNR }\end{array}$ & $\begin{array}{c}\text { Colección Amp. } \\
\text { UNLP }\end{array}$ & \multicolumn{2}{c}{$\begin{array}{c}\text { Colección Los } \\
\text { Zazos UNLP }\end{array}$} & \multicolumn{2}{c}{$\begin{array}{c}\text { Colección EI } \\
\text { Bordo. UNLP }\end{array}$} \\
\hline $\begin{array}{c}\text { Composición } \\
\text { artefactual }\end{array}$ & $\mathrm{n}$ & $\%$ & $\mathrm{n}$ & $\%$ & $\mathrm{n}$ & $\%$ & $\mathrm{n}$ & $\%$ \\
\hline $\begin{array}{l}\text { Otros grupos } \\
\text { tipológicos }\end{array}$ & 27 & 64,28 & 7 & 41,17 & 40 & 15,32 & 8 & 61,53 \\
Bifaces & 3 & 7,16 & 3 & 17,64 & 116 & 44,44 & - & - \\
Esb. Piez. Bif. & 5 & 11,9 & 5 & 29,43 & 51 & 19,54 & 3 & 23,09 \\
Fbas & - & - & - & - & 15 & 5,74 & 2 & 15,38 \\
Unifaces & 2 & 4,76 & - & - & 20 & 7,66 & - & - \\
Artefactos comp. & 5 & 11,9 & 2 & 11,76 & 19 & 7,3 & - & - \\
Total & 42 & 100 & 17 & 100 & 261 & 100 & 13 & 100 \\
\hline
\end{tabular}

Los resultados del análisis tecno-morfológico de las colecciones muestran una abundancia de artefactos formatizados confeccionados con un bajo esfuerzo tecnológico. Aún en los artefactos compuestos, donde uno esperaría una mayor inversión de trabajo como una búsqueda de maximizar la materia prima, encontramos que fueron confeccionados mediante pocos lascados (Figura 3).

En relación a esto último, destacamos que dentro de la clasificación de "otros grupos tipológicos” existen artefactos formatizados enteros con un porcentaje de corteza superior al 50 (Carbonelli 2013b: 243). Estos valores nos indican que en algunos casos, la confección de los instrumentos se efectuó mediante secuencias de reducción cortas, en las cuales no se precisaba quitar toda la corteza de la cara dorsal, o que fueron utilizadas como formas base, lascas externas.

El grupo tipológico más representado es el de los bifaces, seguido por los esbozos de piezas bifaciales, y los artefactos bifaciales con arista sinuosa, produciéndose éstos muchas veces por accidentes de talla, fallos dentro del proceso de reducción, o simplemente descartes o abandonos. Estos instrumentos presentan una menor porción de corteza, relacionada con una mayor intensidad en su producción. 


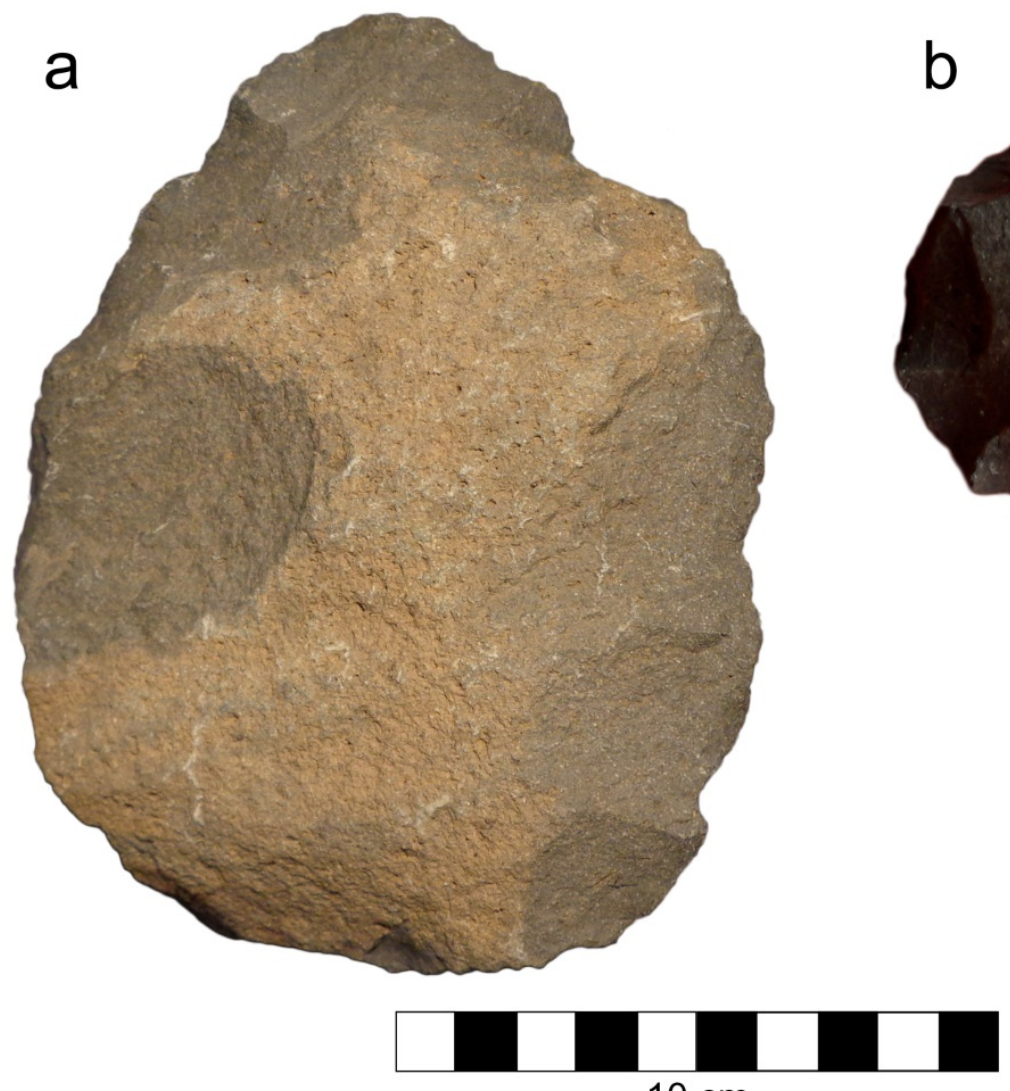

$10 \mathrm{~cm}$
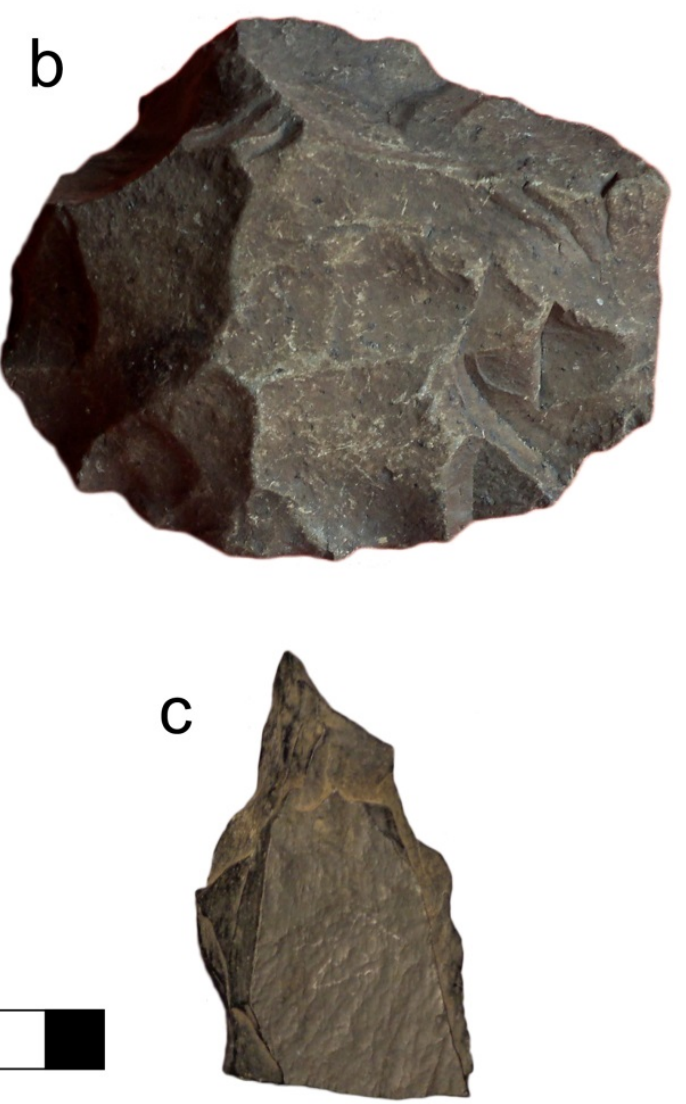

Figura 3. Artefactos formatizados. A. Bifaz parcial. B. Núcleo poliédrico. C. Perforador.

Figure 3. Knapping tools. A. Partial biface. B. Polyhedric core. C. Perforator.

Los únicos artefactos con adelgazamiento bifacial pertenecen a la Colección los Zazos. Se trata de diez bifaces, tres esbozos de piezas bifaciales y un artefacto compuesto, manufacturado sobre un bifaz. El resto de los bifaces presentes en esa y otras colecciones son bifaces parciales, que se encuentran en proceso de producción, donde se ha podido detectar la intención por rebajar el espesor del artefacto y poder generar una sección regular, biconvexa. Estos artefactos formatizados se caracterizan por poseer extensas áreas no formatizadas en algunas de sus caras, con lascados poco profundos en su interior (Hocsman 2006: 98).

La reducción unifacial se concentra básicamente en los unifaces, donde es factible que los artesanos hayan buscado un contorno y una sección específica (Hocsman \& Escola 2007). En el resto de los artefactos formatizados sólo se buscó una morfología particular del filo, mediante una combinación de sectores con lascados profundos y extendidos con otros sectores con formatización sumaria.

\section{Discusión y conclusiones}

En este trabajo se analizaron los conjuntos artefactuales de tres sitios de superficie Ampajango, Los Zazos y El Bordo. Los artefactos de estos sitios provenían en este caso de colecciones de museos. A través del análisis estadístico hemos dado cuenta de la existencia de sesgos en la conformación de las muestras: los primeros trabajos de campo privilegiaron la recolección de artefactos sin barniz del desierto y de artefactos formatizados por encima de otras clases tipológicas.

Una vez reconocidos estos sesgos, decidimos analizar tecno-morfológicamente los artefactos formatizados de las colecciones, porque consideramos que aún era factible obtener información valiosa sobre las estrategias de aprovisionamiento y reducción de materias 
primas líticas. Para una mayor profundidad de dicha temática es necesario vincularla a la estructura regional de los recursos líticos. Según Andrefsky (1994) una variable de suma importancia a la hora de evaluar cómo se encuentra conformado un conjunto instrumental, es la disponibilidad de la materia prima (recursos líticos), los cuales son definidos a través de su abundancia y calidad. Estos factores inciden en la producción de herramientas formales o informales; entre los primeros se encuentran los instrumentos que requieren un esfuerzo adicional en su manufactura, (p.e bifaces) que son estandarizados, factiblemente descartados en lapsos largos; mientras que entre los segundos se encuentran los instrumentos expeditivos, no estandarizados, de retoque sumario (p.e artefactos con retoque marginal o ultramarginal).

A través del registro etnográfico y casos provenientes del trabajo arqueológico, Andrefsky (1994) ha propuesto una relación entre calidad y abundancia de materias primas. Al combinar los resultados de nuestras prospecciones de fuentes potenciales y efectivas de materias primas (Carbonelli 2012; 2013b: 418) con el registro artefactual de las colecciones, podemos concluir que los espacios cantera-taller se encontraban cercanos a zonas con materia prima de calidad buena a regular. A manera de ejemplo, podemos citar el caso de la andesita b o basandesita. Este es de los recursos locales el de mayor calidad por su textura y fractura y se hallaba disponible en las terrazas fluviales (en forma de pequeños nódulos) y en el cauce del río Ampajango, bajo la forma de pequeños rodados. Por otro lado, la andesita g de menor calidad, pero que también presenta ejemplares buenos para la talla, se encuentra plenamente disponible en todas las fuentes secundarias que conforman los ríos que nacen en el Aconquija y en los depósitos de materiales que conforma el glacis donde se encuentra la cantera-taller de Ampajango.

En nuestro análisis de los sitios de superficie del sur del valle de Yocavil, tanto en el sitio Ampajango (Carbonelli 2013a) como en los sitios Los Zazos y el Bordo, hemos observado que en los conjuntos instrumentales existe un predominio de núcleos amorfos; en Amapajango, donde no existió una planificación sobre el tamaño y la forma de la lascas a extraer. Por otra parte el análisis de los conjuntos instrumentales de las colecciones muestra que se hallan conformados en un gran porcentaje por instrumentos informales (artefactos de formatización sumaria, confeccionados mediante pocos lascados) y en menor medida por instrumentos formales (bifaces, unifaces, artefactos compuestos). No obstante, como se desprende de nuestro análisis tecno-morfológico, no existe un gran esfuerzo en la producción de bifaces, ya que muchos de ellos todavía se encuentran en procesos de formatización (bifaces parciales), o son esbozos bifaciales. La presencia de un componente informal en los conjuntos se denota también en la presencia de corteza en la mayoría de los artefactos formatizados, lo que se traduce en secuencias de reducción cortas, sin llegar a la formatización completa de ambas caras.

La gran mayoría de los artefactos formatizados de estos sitios de superficie, fueron confeccionados sobre formas base lascas (Carbonelli 2013b: 174-314), las cuales no presentan similitudes ni en tamaños ni enmódulos de longitud-anchura entre sí. Como primera conclusión, podemos sostener que los conjuntos instrumentales de los sitios de superficie, cantera-taller, situados al sur de Yocavil se corresponden con las expectativas propuestas por Andrefsky (1994), donde en el marco de una estructura regional de abundantes rocas de calidad relativamente baja se manufacturan primordialmente artefactos informales. Por otra parte, es reconocido que los registros de las canteras frecuentemente se encuentran caracterizados por tecnologías mixtas (Beck et al. 2002).

La presencia de bifaces, terminados y en proceso de producción en las colecciones de los museos, puede corresponder al inicio de la manufactura de un toolkit flexible y versátil. Hemos marcado el ínfimo porcentaje de materias primas exóticas en los conjuntos instrumentales analizados. Ante la ausencia de intercambio, es factible que las poblaciones hayan tenido que recorrer grandes distancias para tener acceso a los recursos (Beck \& Jones 
1990). Es necesario para ésto, contar con un conjunto instrumental transportable y flexible compuesto por bifaces, los que posiblemente hayan sido utilizados como núcleos. Dada la estructura regional de recursos líticos, esto es posible dado que "When raw material and adequate sharpness there is no temporal o spatial difference in the location of raw material and the location of stone tool use (...) and we can expect groups living under such circumstances to employ an expedient flake technology, with little use bifaces as cores..." (Kelly 1988: 719).

No obstante, existe una hipótesis alternativa. Basándose en información etnográfica y archivos históricos, Baied \& Somonte (2012) establecen como hipótesis que estos sitios no sólo serían canteras en el sentido estricto, sino espacios destinados a actividades específicas como el aprovechamiento de los recursos vegetales, para los cuales habrían sido necesarios estos instrumentos de gran porte y de filo extendido. Esto conlleva implicancias en lo que respecta a la movilidad, pues dada la abundancia de andesita local (soporte de la mayoría de los artefactos formatizados) y la ausencia de materia prima no local (p.e obsidiana) para este período, Baied y Somonte (2012) consideran que probablemente la movilidad de los cazadores-recolectores de esa región, durante el Holoceno Medio, haya sido limitada a un pequeño rango. Para confirmar cualquiera de las dos hipótesis deben realizarse en el futuro, análisis funcionales de base microscópica que permitan determinar para qué fueron utilizados los bifaces.

A diferencia de lo analizado para los sectores vecinos, en los conjuntos líticos de la cantera-taller Ampajango y de lo que pudimos observar a través de las colecciones de Los Zazos y El Bordo, no registramos reclamación. Hemos observado casos de reciclajes de artefactos en Ampajango y en Los Zazos (Carbonelli 2013b: 212; 283-290), pero que se muestran arqueológicamente contemporáneos. No se detectaron diferencias de barniz entre los primeros lascados de los artefactos y los que provienen de las acciones de reciclaje; teniendo en cuenta que la formación de esta pátina es un proceso de formación lento y que de existir diferencias denotaría claramente este proceso. No obstante, ésta sigue siendo una opción válida a explotar en el futuro. En consonancia con ésto, debemos recordar la presencia de un sesgo en la conformación de las colecciones de los museos: como lo describimos mediante el test estadístico de $\mathrm{Chi}^{2}$, fundamentalmente en la Colección de Ampajango y Los Bordos del Museo de Ciencias Naturales de la UNLP faltan piezas con presencia de barniz. Es dable suponer que si estas colecciones estuviesen completas o estructuradas mediantes recolecciones sistemáticas hubieran aumentado las posibilidades de registrar casos de reclamación. La falta de evidencia de esta actividad nos limita a la hora de establecer lazos y continuidades con poblaciones posteriores, en lo que concierne a la explotación reiterada en el tiempo de un espacio. No obstante, consideramos que hemos dado los primeros pasos para en el futuro poder entender el rol de las canteras-taller al sur del valle de Yocavil con el resto de los espacios de consumo y producción de tecnología lítica.

\section{Agradecimientos}

Este trabajo se realizó en el marco de mi beca posdoctoral CONICET, y con el financiamiento de los subsidios UBACyT GC (2011-2014) y de ANPCYT PICT-2010-2013 (2011-2014).

\section{Referencias}

Andrefsky, W. 1994, Raw-material availability and the organization of technology. American Antiquity, 59 (1): 21-34. (en inglés; in English) ("Disponibilidad de materia prima y la organización de la tecnología”) doi:10.2307/3085499 
Aschero, C. A. 1975, Ensayo para una clasificación morfológica de artefactos líticos aplicados a estudios tipológicos comparativos. Informe presentado al CONICET. Buenos Aires, 113 p. (in Spanish) ("Test for morphological classification of lithic artifacts applied to comparative typological studies”)

Aschero, C. A. 1983, (Revisiones) Ensayo para una clasificación morfológica de artefactos líticos. Apéndices A y B. Cátedra de Ergología y Tecnología. UBA. Informe presentado al CONICET, Buenos Aires, 180 p. (in Spanish) ("Test reviews for morphological classification of lithic artifacts. Appendices A and B”)

Aschero, C. A. \& Hocsman, S. 2004, Revisando cuestiones tipológicas en torno a la clasificación de artefactos bifaciales. In: Temas de arqueología, análisis lítico (Acosta, A., Loponte, D. \& Ramos, M., Eds.), Universidad Nacional de Luján, Luján: p. 7-25. (in Spanish) ("Reviewing typological issues surrounding the classification of bifacial artifacts”)

Baied, C. \& Somonte, C. 2012, Mid-Holocene geochronology, palaeoenvironments, and occupational dynamics at Quebrada de Amaicha, Tucuman, Argentina. Quaternary International, 299: 80-89. (en inglés; in English) (“Geocronología de Holoceno medio, paleoambientes y la dinámica de ocupacional en la Quebrada de Amaicha, Tucumán, Argentina”) doi:10.1016/j.quaint.2012.11.025

Beck, C. \& Jones, G. 1990, Toolstone Selection and Lithic Technology in Early Great Basin Prehistory. Journal of Field Archaeology, 17: 283-299. (en inglés; in English) ("La selección de piedra para las herramientas y la tecnología lítica en la prehistoria temprana de la Great Basin”) doi:10.2307/530023

Beck, C., A., Taylor, G., Jones, C., Fadem, C., Cook, S., Millward. 2002, Rocks are heavy: Transport cost and Paleoarchaic quarry behavior in the Great Basin. Journal of Anthropological Archaeology, 21: 481-507. (en inglés; in English) ("Las rocas son pesados: El costo del transporte y el comportamiento de cantera Paleoarcaico en la Great Basin”) doi:10.1016/S0278-4165(02)00007-7

Bellelli, C. 2005, Tecnología y materias primas a la sombra de Don Segundo. Una canterataller en el valle de Piedra Parada. Intersecciones en Antropología, 6: 75-92. (in Spanish) ("Technology and raw materials in the shadow of Don Segundo. A quarry workshop in the valley of Piedra Parada").

URL: http://www.scielo.org.ar/scielo.php?pid=S1850373X2005000100007\&script=sci_arttext

Carbonelli, J.P. 2011, “Motivos porque y para” en la tecnología lítica de un sitio formativo en el Valle de Yocavil, provincia de Catamarca. Intersecciones en Antropología, 12: 31-45. (in Spanish) ("Reasons for and" lithic technology in a training site in the Valley of Yocavil province of Catamarca").

URL: http://www.scielo.org.ar/scielo.php?pid=S1850373X2011000100003\&script=sci_arttext

Carbonelli, J.P. 2012, Base regional de recursos líticos y fuentes de materias primas al sur del Valle de Yocavil. Estudios Sociales del NOA, 12: 111-137. (in Spanish) ("Regional basis of lithic resources and raw materials sources of south Yocavil Valley"). URL: http://revistascientificas.filo.uba.ar/index.php/esnoa/article/view/787

Carbonelli, J.P. 2013a, El sitio Ampajango, cincuenta años después. Arqueología, 19 (1): 4164. (in Spanish) (“The Ampajango site, fifty years later”).

URL: https://sites.google.com/site/reviarqueol3/home/arqueologia-19 
Carbonelli, J.P. 2013b, Técnicas líticas en paisajes cazadores y agropastoriles al sur del valle de Yocavil. Doctoral Thesis at the Faculty of Philosophy and Letters, Universidad de Buenos Aires, Buenos Aires. 502 p. (in Spanish) ("Lithic techniques in hunter and agropastoralist landscapes in the south of the Yocavil Valley")

Church, T. 1994, Terms in Lithic Resource Studies: Or, is this a Lateritic Silcrete or a Ferruginous Woodgrained Chert? In: Lithic Resource Studies: A Sourcebook for Archaeologists (Church, T., Ed.), Lithic Technology Special Publication Vol. 3, Department of Anthropology, University of Tulsa, Tulsa: p. 9-25. (in en inglés; in English) ("Terminología en el estudio de los recursos líticos: O, este es un silcrete lateríticos o una sílex ferruginosa con grano de madera?")

Cigliano, E. 1961, Noticia sobre una nueva industria precerámica en el Valle de Santa María (Catamarca): El Ampajanguense. Anales de Arqueología y Etnología, 16: 169-179. (in Spanish) ("News about a new pre-ceramic industry in the Valle de Santa María (Catamarca): The Ampajanguense”)

Cigliano, E. 1964, El precerámico en el N. W. Argentino. Separata de Arqueología de Chile Central y áreas vecinas, 1: 191-196. (in Spanish) ("The preceramic in N. W. of Argentina”)

Cigliano, E. 1968, Panorama general de las industrias precerámicas en el noroeste argentino. Actas del XXXVII Congreso Internacional de Americanistas, 3: 339-344. (in Spanish) ("Overview of preceramic industries in northwestern Argentina”)

Cigliano, E., Bereterbide, S., Carnevali, B., Lorandi, A.M. \& Tarragó, M. N. 1962, El Ampajanguense. Publicación del Instituto de Antropología, 5: 7-104. (in Spanish) ("The Ampajanguense")

Civalero, M.T. \& Franco, N.V. 2003, Early human Ocuppation at the West of Santa Cruz province, Southern End of South America. Quaternary Internacional, 109-110: 77-86. (en inglés; in English) ("La ocupación humana temprana en el oeste de la provincia de Santa Cruz, la extensión meridional de América del Sur”) doi:10.1016/S10406182(02)00204-5

Durando, P. M. García Salemi \& G. Platanía. 1986, Estudios geomorfológicos; paleoclimas, dataciones relativas y tipología a partir de paleolitos del Valle de Santa María (Provincias de Catamarca y Tucumán, República Argentina). Actas y Trabajos del VI Congreso Peruano: Hombre y Cultura Andina, 6: 89-109. (in Spanish).

(“Geomorphological studies; paleoclimates, relative dating and typology from paleolitos del Valle de Santa María (provinces of Catamarca and Tucuman, Argentina)”)

Elías, A. 2010, Estrategias tecnológicas y variabilidad de los conjuntos líticos de las sociedades tardías en Antofagasta de la Sierra (Provincia de Catamarca, Puna Meridional Argentina). Doctoral Thesis at the Faculty of Philosophy and Letters, Universidad de Buenos Aires, Buenos Aires. 541 p. (in Spanish) ("Technological strategies and variability of lithic assemblages of late societies in Antofagasta de la Sierra (Province of Catamarca, Argentina Southern Puna”)

Escola, P. 2000, Tecnología lítica y sociedades agropastoriles tempranas. Doctoral Thesis at the Faculty of Philosophy and Letters, Universidad de Buenos Aires, Buenos Aires. 430 p. (in Spanish) (“Lithic technology and early agropastoral societies”) 
García Salemi, M. \& Durando, P. 1985, Sobre cronologías y paleoclimas en la Quebrada de Amaicha. Centro Estudio Regiones Secas, 2(2): 1-4. (in Spanish) (“About chronology and paleoclimate in the Quebrada de Amaicha”)

Hocsman, S. 2006, Producción lítica, variabilidad y cambio en Antofagasta de la Sierra -ca. 5500-1500 AP. Doctoral Thesis at the Faculty of Natural Sciences and Museum, Universidad Nacional de La Plata, Argentina. 532 p. (in Spanish) ("Lithic production, variability and change in Antofagasta de la Sierra -ca. 5500-1500 AP”)

Hocsman, S. \& Escola, P, 2007, Inversión de trabajo y diseño en contextos líticos agropastoriles (Antofagasta de la Sierra, Catamarca). Cuadernos del Instituto Nacional de Antropología y Pensamiento Latinoaericano, 21(2006-2007): 75-90. (in Spanish) ("Investment design work and agro-pastoral contexts lithic, Antofagasta de la Sierra, Catamarca”)

Kelly, R. 1988, The three sides of a biface. American Antiquity, 53 (4): 717-734. (en inglés; in English) ("Los tres lados de un biface") doi:10.2307/281115

Nami, H. 1992, El subsistema tecnológico de la confección de instrumentos líticos y la explotación de los recursos del ambiente: una nueva vía de aproximación. Shincal 2: 2253. (in Spanish). ("Technological subsystem making stone tools and exploiting the resources of the environment: a new avenue of approach").

Paulides, L. 2006, El núcleo de la cuestión. El análisis de los núcleos en los conjuntos líticos. In: El modo de hacer las cosas. Artefactos y ecofactos en Arqueología, (Pérez de Micou, C., Ed.), Universidad de Buenos Aires, Buenos Aires: p. 67-101. (in Spanish) ("The core of the matter. Analysis of cores in lithic assemblages")

Ruiz Huidobro, O. 1972, Descripción Geológica de la Hoja 11E Santa María, provincias de Catamarca y Tucumán: Carta geológico-económica de la República Argentina, escala 1:200.000. Boletin Vol. 134. Dirección Nacional de Minería, Servicio Nacional Minero Geológico, Buenos Aires. 64 p. (in Spanish) (“Geological description of sheet 11E, Santa Maria, provinces of Catamarca and Tucuman: Geological-economic map of the Argentine Republic, scale 1:200,000”)

Spano, R. 2011, Primera sistematización de las características estilísticas de la alfarería fina del sitio Soria 2 (Valle de Yocavil, Noroeste Argentino). Revista del Museo de Antropología, 4: 127-144. (in Spanish) ("First systematisation of the stylistic characteristics of fine pottery of the Soria 2 site (Yocavil Valley, Northwest Argentina)”) URL: http://revistas.unc.edu.ar/index.php/antropologia/article/view/5492/

Somonte, C. 2009, Tecnología lítica en espacios persistentes de Amaicha del Valle (Tucumán). Doctoral Thesis at the Faculty of Philosophy and Letters, Universidad de Buenos Aires, Buenos Aires. 395 p. (in Spanish) ("Lithic technology in persistent spaces of Amaicha del Valle, Tucumán”)

Somonte, C. \& C. Baied. 2011, Recursos líticos, aprovisionamiento y aspectos temporales de fuentes de abastecimiento en Amaicha del Valle, Tucumán, Argentina.

Comenchingonia, 14: 97-113. (in Spanish) ("Lithic resources, procurement and temporal aspects of sourcing in Amaicha del Valle, Tucumán, Argentina”).

URL: http://www.comechingonia.com/COMECHINGONIA\%20IMPRESA/comeching onia_N14.htm

Somonte, C. \& C. Baied. 2013, Edad mínima de exposición de superficies en canteras-taller: reflexiones en torno a las primeras dataciones mediante microlaminaciones del barniz 
de las rocas (VML) para el noroeste argentino. Chungara, 45 (3): 427-445. (in Spanish) ("Minimum surface exposure age of stone quarry - workshops: insights on the first rock-varnish microlamination (vml) dates for northewestern Argentina”). doi:10.4067/S0717-73562013000300005

Valverde, Federico 2003, Análisis de los desechos líticos de la ocupación inicial del sitio cueva Tixi (Provincia de Buenos Aires): cadena operativa de producción y técnicas de talla tempranas. Relaciones de la Sociedad Argentina de Antropología, 28: 185-202. (in Spanish) ("Analysis of lithic debris from the initial occupation of the cave site Tixi (Buenos Aires): operational chain of production and early carving techniques.

URL: http://www.saantropologia.com.ar//wpcontent/uploads/2015/01/Relaciones\%2028/28\%20Valverde.pdf 


\title{
Contribution to the analysis of collection procurement mechanisms: The case of the quarry workshop sites of Yocavil Valley (Northwest Argentina)
}

\author{
Juan Pablo Carbonelli \\ CONICET. Museo Etnográfico, Universidad de Buenos Aires (UBA), Estados Unidos $33873^{\circ}$ A Lanús Oeste, \\ Argentina. Email: juanp.carbonelli@gmail.com
}

\begin{abstract}
:
In this paper we propose to analyze and compare four collections of artifacts, which come from surface sites located south of the Calchaquíes Valleys. The quarry-workshop sites of Ampajango, El Bordo and Los Zazos have been previously studied and some of their assemblages are in the Museum of Natural Sciences of the National University of La Plata and in the Museum of Anthropology of the National University of Rosario, both in Argentina. These sites had been characterized as the first hunter-gatherer occupations in the valley of Yocavil (Cigliano 1964; 1968), mainly comprised of bifaces, scrapers and cores, most of them covered by a particular rock coating (rock varnish), which is desert varnish.

In this paper we offer a revision and a new interpretation of such artifact assemblages.

The questions that guide our work are: Will a new analysis of these collections allow us to observe complex processes such as reuse-recycling? What role did these spaces have at a regional level in sourcing decisions and exploitation of raw materials?

By looking at the collections we have detected two problems. First, a greater presence of desert varnish on the parts collected during our fieldwork (Carbonelli 2013) in comparison with the ones in the above named collections. This variable is particularly relevant because a greater number of artifacts with varnish present are more likely to reveal cases of reuse-recycling; that is to say temporal difference between different sets of flaked stone. To solve this problem, we conducted a $\mathrm{Chi}^{2}$ analysis in order to test the null hypothesis of the absence of a difference between the proportions of artifacts with varnish from our collections and museum collections.

Secondly, we detected an existing imbalance between the high frequency of knapping tools in detriment of debitage and cores in museum collections. In order to quantitatively address the diversity of each set, we compared the typological classes of each of the matching sets. Diversity is a way of measuring the variation since it describes attributes of a population composed of classes (attributes) that vary in frequency (wealth), which allows us to discuss structural issues of the sample. To explore the richness we use indicators that are not too sensitive to sample size, such as the Simpson Dominance Index and Eveness.

From the methodological aspect, statistical analysis allowed us to recognize the biases that built the samples. The $\mathrm{Chi}^{2}$ tests indicate to us that there is a lower proportion of artifacts with desert varnish in the collections than what would fit reality. This manifests mainly in the absence of cases of reuse-recycling, evident in the differences of varnish between flaked artifacts. Moreover, the analysis of diversity warns us that there is an over-representation of finished tool type artifacts in collections, which makes difficult the description of the production sequence. Based on these biases, through techno-morphological analysis we characterized the lithic production of quarry-workshop sites. Generally speaking, they present an expeditious technology comprises of amorphous cores and partial bifaces, which were then used as cores.
\end{abstract}


Even in the case of bifaces, there was no great effort put into their production, since many of them were still in the process of being knapped. The presence of an informal component in sets are also denoted by the presence of cortex in most knapped artifacts resulting in sequences of short reductions, without achieving a complete knapping of both sides.

This low knapping of the sets is explained if we combine these observations with the results of our survey of potential and effective sources of raw materials (Carbonelli 2012, 2013b). The pieces of the collections come from places close to areas of good to fair quality raw materials, so expeditious technology with a small holding of bifaces and cores is consistent.

Keywords: Yocavil Valley; lihic quarry; collections; hunter-gatherers; bifaces 